\title{
Trying Times and Trying Out Solutions: Intimate Partner Violence Screening and Support for Women Veterans During COVID-19
}

\author{
Fernanda S. Rossi, $P h D^{1,2}$ (D), Megha Shankar, $M D^{1,2}$, Kelly Buckholdt, $P h D^{3}$, \\ Yuki Bailey, $M A^{4}$, Sonoo Thadaney Israni, MBA4, and Katherine M. Iverson, PhD $D^{5,6}$ \\ ${ }^{1}$ VA Palo Alto Health Care System Center for Innovation to Implementation (Ci2i), Menlo Park, CA, USA; ${ }^{2}$ Center for Primary Care and Outcomes \\ Research (PCOR) and Center for Health Policy (CHP), Stanford University, Stanford, CA, USA; ${ }^{3}$ National Intimate Partner Violence Assistance \\ Program, Veterans Health Administration, Washington, DC, USA; ${ }^{4}$ Presence Center, Stanford University School of Medicine, Stanford, CA, USA; \\ ${ }^{5}$ Women's Health Sciences Division, National Center for PTSD, VA Boston Healthcare System, Boston, MA, USA; ${ }^{6}$ Department of Psychiatry, Boston \\ University School of Medicine, Boston, MA, USA.
}

Initial reports indicate widespread increases in intimate partner violence (IPV) rates during the coronavirus disease 2019 (COVID-19) pandemic. Women veterans are at particular risk for experiencing IPV, and the COVID-19 pandemic and resulting stay-at-home orders may be exacerbating this risk. IPV screening and intervention are an integral part of the care provided to women veterans in the Veteran's Health Administration (VHA). Current changes in healthcare delivery during COVID-19 may present challenges to the VHA's standard methods of initiating IPV screening and intervention with women veterans. We discuss the potential challenges VHA healthcare providers may be encountering when conducting routine IPV screening during the COVID-19 pandemic and when providing resources and support to women veterans experiencing IPV. We describe solutions to these challenges, including existing efforts led by the VHA IPV Assistance Program (IPVAP) as well as additional potential solutions. New ideas and partnerships will be critical for helping the VHA continue to assist women veterans experiencing IPV as the COVID-19 pandemic evolves. Though our focus is on women veterans and the VHA, the challenges and solutions we discuss are likely applicable to other populations experiencing IPV and other health care systems screening for IPV.

KEY WORDS: intimate partner violence; women veterans; COVID-19; screening; pandemic.

J Gen Intern Med 35(9):2728-31 DOI: $10.1007 / \mathrm{s} 11606-020-05990-0$

(c) Society ofGeneral InternalMedicine (This is aU.S. government work and not under copyright protection in the U.S.; foreign copyright protection may apply) 2020

I ntimate partner violence (IPV), defined as physical or sexual violence, stalking, psychological aggression, or coercion by a past or current intimate partner, ${ }^{1}$ is an all too

Received May 18, 2020

Accepted June 12, 2020

Published online June 30, 2020 common experience in the lives of women. Women veterans may be at particular risk ${ }^{2}$ as up to $60 \%$ of women veterans in relationships report experiencing IPV. ${ }^{3}$ The burden of IPV on women veterans is often compounded with experiences such as sexual assault and harassment during military service. ${ }^{4,5}$

In 2019, the Veteran's Health Administration (VHA) issued a national directive calling for routine IPV screening and provision of intervention across VHA facilities. ${ }^{6}$ IPV screening and intervention are necessary for maintaining women veteran safety and, thus, an integral part of the care provided to women by the VHA. As with other populations, the spread of the coronavirus disease 2019 (COVID-19) has resulted in unprecedented disruptions and changes to veteran health care, and stay-at-home orders may put women veterans at even higher risk of IPV. Current changes in healthcare delivery during COVID-19 may present challenges to the VHA's standard methods of initiating IPV screening and intervention with women whose lives are at increased risk of danger due to IPV.

This is particularly concerning during a time when IPV rates could be increasing for women veterans, given increased emotional, financial, occupational, and social stressors due to the COVID-19 pandemic. Initial reports in China indicate a threefold increase in IPV-related reports to the police during February of 2020 compared with February of $2019 .^{7}$ There is anecdotal evidence of similar increases in IPV rates during the COVID19 pandemic across Brazil, Australia, Great Britain, and the USA. $^{8,} 9$ The effects of the pandemic on IPV rates may be long-lasting. For example, unemployment resulting from the pandemic may keep abusive partners at home for extended periods of time, further entrapping women. Partners may also constantly feel on edge due to occupational and financial stressors, possibly triggering greater use of violence against women.

We discuss two critical areas in which the COVID-19 pandemic may create challenges in the VHA's provision of IPV-related services and for which creative solutions are being utilized: (1) routine IPV screening and (2) providing resources and support to women experiencing IPV. Though our focus is on women veterans and the VHA, the challenges and solutions we discuss are likely applicable to 
other populations experiencing IPV and other health care systems screening for IPV.

\section{CHALLENGES WITH SCREENING}

The COVID-19 pandemic and the associated stay-at-home orders implemented to reduce spread of the virus may pose challenges to conducting IPV screening in the VHA. A large proportion of IPV screening in the VHA is conducted inperson at primary and specialty care clinics (e.g., mental health clinics). However, given widespread stay-at-home orders, many non-essential health care visits have either been canceled or are being completed using telehealth (i.e., phone, VA Video Connect, clinical video telehealth). IPV screening conducted via telehealth could help identify women veterans experiencing IPV but, without appropriate consideration and provider practices, may inadvertently put them at even greater danger, as a violent or controlling partner may overhear discussions regarding IPV, unexpectedly walk into the room, or use technological abuse strategies for monitoring (e.g., recording calls). Upon hearing discussion of relationship health (even without disclosure of IPV incidents), a partner may become suspicious or angry and could use abusive or violent behavior. Controlling partners may prevent women from finding isolated locations inside or outside the home in which to speak privately, and attempts to seek privacy may be met with suspicion or aggression (i.e., "Why do you need to talk in private?" "What are you going to talk about?"). Also, due to stay-at-home orders, which have led to limited community access, women experiencing IPV may have significant difficulties finding safe locations where they are able to freely seek assistance with IPV. For such reasons, it is particularly difficult to ensure the environment is private and safe, a prerequisite for inquiring about IPV experiences. Unlike in-person office visits, where providers should ensure no partner, family member, or child over the age of 2 is present when engaging in IPV screening, telehealth necessitates assuming that someone else may overhear even if not in view. As a result, providers have additional complexity to consider before screening for IPV or providing necessary support so as not to put women veterans at greater risk of danger. Without creative solutions, these privacy and safety-related concerns could reduce opportunities for providers to ask about IPV as well as for patients to disclose IPV. Reductions in patient disclosure opportunities in turn make it more challenging for women experiencing IPV to obtain information about IPV-related resources and services (e.g., medical, mental health, social work, financial, legal, shelter, and housing resources).

\section{CHALLENGES IN PROVIDING SUPPORT}

Though stay-at-home orders are described as essential for helping slow the spread of COVID-19, ${ }^{10}$ associated closures can limit resources available to women experiencing IPV. For example, domestic violence shelters, court houses, and other public service agencies may be closed or limited to virtual platforms, further reducing space for privacy and safety. Even the ability to utilize crisis hotlines may be hindered if women are unable to find a safe location for calls or text messaging. Safety plans that were previously developed may no longer be as applicable if friends, family, or shelters are not an option for women fleeing an abusive relationship. Reaching healthcare providers to ask for assistance and obtain resources may be challenging when access to in-person appointments is limited and/or when telehealth options are limited due to difficulties accessing a safe location for contact. Additionally, fear of exposure to COVID-19 may contribute to reluctance to access medical care, including going to an emergency department in the case of IPV-related injury. Even when providers can screen for IPV, their ability to intervene effectively may be limited if they rely on knowledge of pre-COVID-19 IPV resources and supports, since such resources may no longer be available for women at the same levels as they were prior to the pandemic. Reduced ability to provide available resources to women experiencing IPV is a critical issue, particularly during a time in which women may be experiencing an increased need for help.

\section{INITIAL SOLUTIONS}

\section{Current VHA Efforts}

The VHA, the largest integrated health care system in the USA, offers IPV assistance and prevention services through the Intimate Partner Violence Assistance Program (IPVAP). Through the IPVAP, the VHA has already taken numerous steps to address challenges with IPV screening and the provision of resources. On a national and local level, the IPVAP is a comprehensive person-centered, recovery-oriented, and trauma-informed program committed to helping veterans, their partners, and employees impacted by IPV. During the COVID-19 pandemic, the IPVAP has been promptly reviewing, adapting, and creating IPV guidance and resources to fit the current situation and associated training needs, as well as disseminating critical information to VHA providers and women veterans. The rapid response during this time of global challenge occurred through the combined efforts of national IPVAP leadership, a dedicated network of IPVAP Coordinators on staff at VHA facilities nationwide, and in partnership with stakeholders within and outside the VHA who are dedicated to fostering relationship health and safety for women veterans.

Addressing Challenges with IPV Screening. To address potential challenges with IPV screening, the IPVAP recommends that providers continue to follow the established IPVAP trauma-informed screening protocol, with 
guidance for how to adapt the environmental safety check for the telehealth context. Specifically, screeners should perform an environmental safety check to ensure a partner or other individuals are not present before starting a virtual visit by asking simple yes-or-no questions, which when using visual technology can be responded to with a head nod. If the partner unexpectedly walks into the room, the provider should wait quietly. Additionally, if the patient is wearing headphones, the provider may ask yes-or-no questions, such as "Do you need to hang up?", "Are you safe?", or "Should I send 911?". Such virtual care considerations can enable safe opportunities to discuss IPV-related information, while also reducing risk of danger.

Overcoming Challenges in Providing IPV Support. To help overcome challenges accessing care and the ability to provide resources, the IPVAP developed a multi-method approach to raise awareness and facilitate access to services through social media platforms, internal e-mails, and staff- and veteranspecific fact sheets. ${ }^{11,12}$ These multi-faceted strategies provide education about increased risk and available resources across internal and external programs, as well as provide multiple means of reaching women veterans so that, when they are in a safe place to reach out for assistance, they have multiple options and up-to-date contact information. Materials include social media images and posts, educational content and contact information, links to the IPVAP public-facing website, a roster of IPVAP contacts by state, and community partners, such as the National Domestic Violence Hotline. To reduce risk associated with accessing IPV-related content online (e.g., if a partner walks into the room or accesses web history), the IPVAP website (https://www.socialwork.va.gov/ IPV/) has a "quick escape" button which redirects to the va. gov homepage. The website also includes links to external stakeholder organizations with COVID-19 specific information and similar practices for reducing risk. IPVAP Coordinators at VHA facilities nationally routinely update their local resource and referral information (e.g., shelters that are currently accepting women) and disseminate the information to interprofessional staff who may screen for IPV and/or have an opportunity to provide resources to someone in need. Additional resources shared by IPVAP include self-directed safety planning tools, such as myPlan, a smartphone application that assists those experiencing IPV to develop individualized safety plans. ${ }^{13}$

For women veterans who are able to safely engage in telehealth, many VHA IPV-specific services remain available. For example, they can have telehealth interactions with an IPVAP Coordinator or Champion (e.g., social worker or mental health provider trained to respond to IPV using best practices) for assessment, safety planning, and to access resources and interventions. Supported by the IPVAP, several VHA facilities are using telehealth to offer Recovering from IPV through Strengths and Empowerment (RISE), ${ }^{14}$ a patientcentered and trauma-informed brief counseling intervention for women experiencing IPV. Likewise, many facilities are continuing to offer Strength at Home, an evidence-based intervention for reducing use of violence in intimate relationships, by implementing telehealth when possible. ${ }^{15}$ Women veterans may also use telehealth for mental health services and to engage with a primary care provider and receive routine health services that may directly or indirectly reveal IPV experience. Discussion around reproductive health, for example, may reveal instances of abuse, particularly since pregnancy is associated with increased risk of IPV. ${ }^{16}$

Additional Potential Solutions. While the VHA has taken numerous steps to assist women veterans experiencing IPV, additional solutions will need to be developed as the pandemic evolves. For example, it may be critical to establish VHA secure messaging for IPV screening and resource provision that women veterans can access when it is safe to do so. For those without access to a computer or phone, it may be necessary to discreetly place informational brochures at grocery stores or other locations that provide essential services. It may also be important to partner with media outlets to increase dissemination of resources and social media messaging developed by the VHA. Additionally, continued coordination and partnerships between internal VHA programs (e.g., IPVAP, Women's Health, Mental Health) and external programs (e.g., shelters, advocacy groups) will be necessary so that after the pandemic coordination and joint efforts continue to benefit women.

\section{CONCLUSION}

Overall, new ideas and partnerships will be critical for helping the VHA continue to assist women veterans experiencing IPV during the COVID-19 pandemic and future public health emergencies. This is a particularly trying time for women veterans impacted by IPV, and we must remain dedicated to rapidly developing and implementing novel solutions that can protect their safety. We serve, because she served.

Corresponding Author: Fernanda S. Rossi, PhD; VA Palo Alto Health Care System Center for Innovation to Implementation (Ci2i), Menlo Park, CA, USA (e-mail: fsrossi@stanford.edu).

Funding Information This article is supported in part by the VA Office of Academic Affiliations and Health Services Research and Development Service Research funds.

\section{Compliance with Ethical Standards:}

Conflict of Interest: The authors declare that they do not have a conflict of interest.

Disclaimer: The views expressed in this article are those of the authors and do not necessarily reflect the position or policy of the Department of Veterans Affairs or the United States Government. 


\section{REFERENCES}

1. Breiding MJ, Basile KC, Smith SG, Black MC, Mahendra R. Intimate partner violence surveillance uniform definitions and recommended data elements. Centers for Disease Control and Prevention National Center for Injury Prevention and Control. 2017. Available at: https://www.cdc.gov/ violenceprevention/pdf/ipv/intimatepartnerviolence.pdf. Accessed May 17, 2020.

2. Dichter ME, Cerulli C, Bossarte RM. Intimate partner violence victimization among women veterans and associated heart health risks. Womens Health Issues. 2011;21(4, Supplement):S190-S194; doi:https://doi.org/10.1016/j.whi.2011.04.008

3. Iverson KM, Vogt $\mathbf{D}$, Maskin RM, Smith BN. Intimate partner violence victimization and associated implications for health and functioning among male and female post-9/11 veterans. Med Care. 2017;55:S78; doi:https://doi.org/10.1097/MLR.0000000000000741

4. Dichter ME, Haywood TN, Butler AE, Bellamy SL, Iverson KM Intimate partner violence screening in the veterans health administration: Demographic and military service characteristics. Am J Prev Med. 2017;52(6):761-768; doi:https://doi.org/10.1016/j.amepre.2017.01.003

5. Kimerling R, Iverson KM, Dichter ME, Rodriguez AL, Wong A, Pavao J. Prevalence of intimate partner violence among women veterans who utilize veterans health administration primary care. J Gen Intern Med. 2016;31(8):888-894; doi:https://doi.org/10.1007/s11606-016-3701-7

6. VHA Directive 1198. Intimate Partner Violence Assistance Program. 2019. Available at: https://www.google.com/url? $\mathrm{sa}=\mathrm{t} \& \mathrm{rct}=\mathrm{j} \& \mathrm{q}=\& \mathrm{esrc}=\mathrm{s} \&$ source=web\&cd=1\&ved=2ahUKEwiQm5jezrvpAhVmIDQIHUCEDWcQFjAAegQIBRAB\&url=https\%3A\%2F\%2Fwww.va. gov\% $2 \mathrm{Fvhapublications} \% 2 \mathrm{FViewPublication}$. asp $\% 3 \mathrm{Fpub}$ ID\%3D8192\&usg=AOvVaw2ulow2C47bi53GOA_i7ESP. Accessed April $22,2020$.

7. Wanqing $\mathbf{Z}$. Domestic violence cases surge during COVID-19 epidemic. 2020. Available at: https://www.sixthtone.com/news/1005253/domestic-violence-cases-surge-during-covid-19-epidemic. Accessed March 28, 2020

8. Ott H. As Domestic violence surges, BBC journalist anchors news with hotline written on her hand. CBS News. 2020. Available at: https://www. cbsnews.com/news/domestic-violence-uk-coronavirus-bbc-journalistvictoria-derbyshire-anchors-news-hotline-number-hand/. Accessed April 23, 2020.

9. Peterman A, Potts A, O'Donnell M, et al. Pandemics and violence against women and children. Center for Global Development. 2020 Available at: http://iawmh.org/wp-content/uploads/2020/04/pandemics-and-vawg-april2.pdf. Accessed April 23, 2020.

10. CDC. Coronavirus Disease 2019 (COVID-19). Centers for Disease Control and Prevention. 2020. Available at: https://www.cdc.gov/coronavirus/ 2019-ncov/prevent-getting-sick/social-distancing.html. Accessed May 1, 2020.

11. Intimate Partner Violence Assistance Program. Intimate partner violence and Novel Coronavirus Disease (COVID-19) response. Veterans Health Administration. 2020. Available at: https://www.socialwork.va.gov/IPV/ docs/COVID19-IPV-Staff-Factsheet_Final.pdf. Accessed May 1, 2020.

12. Intimate Partner Violence Assistance Program. Staying safe during COVID-19. Veterans Health Administration. 2020. Available at: https:// www.socialwork.va.gov/IPV/docs/COVID19-IPV-Veteran-Factsheet_Final.pdf. Accessed May 1, 2020.

13. Glass NE, Perrin NA, Hanson GC, et al. The Longitudinal impact of an internet safety decision aid for abused women. Am J Prev Med. 2017;52(5):606-615; doi:https://doi.org/10.1016/j.amepre.2016.12.014

14. Iverson KM, Gregor K, Gerber MR. RISE: Recovering from IPV through Strengths and Empowerment. A treatment manual. 2019.

15. Taft CT, Macdonald A, Creech SK, Monson CM, Murphy CM. A randomized controlled clinical trial of the strength at home men's program for partner violence in military veterans. J Clin Psychiatry. 2016;77(9):1168-1175; doi:https://doi.org/10.4088/JCP.15m10020

16. Jasinski JL. Pregnancy and domestic violence: A review of the literature. Trauma Violence Abuse. 2004;5(1):47-64; doi:https://doi.org/10.1177/ 1524838003259322

Publisher's Note: Springer Nature remains neutral with regard to jurisdictional claims in published maps and institutional affiliations. 